\title{
ON THE COHOMOLOGY GROUPS OF A POLARISATION AND DIAGONAL QUANTISATION(1)
}

\author{
BY
}

\author{
J. H. RAWNSLEY
}

\begin{abstract}
The sheaf $\delta_{F}(L)$ of germs of sections of a line bundle $L$ on a manifold $X$ covariant constant with respect to a flat connection defined for vectors in a complex subbundle $F$ of the tangent bundle has a resolution by differential forms defined on $F$ with values in $L$ provided $F$ satisfies the integrability conditions of the complex Frobenius theorem. This includes as special cases the de Rham and Dolbeault resolutions.
\end{abstract}

If there is a free $S^{1}$ action on $X$ whose generator is tangent to $F$, let $Y$ be the subset of $X$ where parallel transport in $L$ around the $S^{1}$ orbits is trivial. It is shown that the cohomology groups of $\delta_{F}(L)$ depend only on the restriction of $\delta_{F}(L)$ to $Y$. This is used to obtain a spectrum for a periodic Hamiltonian flow with generator in a polarisation. In the case of a classical harmonic oscillator this spectrum is found to be the same as that of the quantum mechanical oscillator.

1. Introduction. In [6], [8], and [22] B. Kostant and J.-M. Souriau independently developed a theory of geometric quantisation. One seeks to associate differential operators to functions on a symplectic manifold so as to preserve as much as possible of the Poisson bracket structure of the functions. Such a theory was also considered in [11]-[13] by E. Onofri and M. Pauri. For further details see also [1], [14], [15], [18], [19].

The following structure is required: If $X$ is the manifold with symplectic form $\omega$, one requires a line bundle $L^{\omega}$ over $X$ having a connection $\nabla^{\omega}$ whose curvature is $2 \pi i \omega$ and with a parallel transport invariant Hermitian structure (often $\omega$ is said to be the curvature of $\nabla^{\omega}$, whilst $R$. Blattner [1] introduces also a constant $h$ into these definitions). A polarisation of $(X, \omega)$ is a maximally isotropic involutive complex subtangent bundle $F$. If $N_{F}^{1 / 2}$ denotes the bundle

Received by the editors January 30, 1976.

AMS (MOS) subject classifications (1970). Primary 58A10, 58A30, 58F05; Secondary 55B30.

Key words and phrases. Line bundle, integrable tangent subbundle, sheaf of covariant constant sections, Poincaré lemma, resolution, periodic Hamiltonian flow, quantisation, Bohr-Sommerfeld condition.

(') Research supported by the Sonderforschungsbereich 40, Bonn. 
of $\frac{1}{2}$-forms normal to $F$ and $L=L^{\omega} \otimes N_{F}^{1 / 2}, L$ has an $F$-connection (see $\S 4$ ), and one denotes by $\delta_{F}$ the sheaf of germs of sections of $L$ covariant constant along $F, S_{F}=\Gamma\left(\delta_{F}\right)$ is the space of polarised sections.

If $\varphi$ is a smooth function on $X$, $\xi_{\varphi}$ denotes the Hamiltonian vector field associated to $\varphi$. An operator $\delta(\varphi)$ is defined on $\Gamma\left(L^{\omega}\right)$ by $\delta(\varphi)=\nabla_{\xi_{\varphi}}^{\omega}+2 \pi i \varphi$ and satisfies $\delta([\varphi, \psi])=\delta(\varphi) \delta(\psi)-\delta(\psi) \delta(\varphi)$ where the Poisson bracket $[\varphi, \psi]$ equals $\xi_{\varphi}(\psi)$. Let $C_{F}^{0}$ be all functions $\varphi$ with $\xi_{\varphi}$ a section of $F$ and $C_{F}^{1}$ all functions whose Hamiltonian vector fields are infinitesimal automorphisms of $F$; then $C_{F}^{1}$ is a Lie algebra under Poisson bracket, $C_{F}^{0}$ is an abelian ideal in $C_{F}^{1}$, and if $\varphi$ is in $C_{F}^{1}$ there is a natural Lie derivative action of $\xi_{\varphi}$ in $\Gamma\left(N_{F}^{1 / 2}\right)$. Combining $\delta(\varphi)$ with this Lie derivative gives a differential operator $\delta_{F}(\varphi)$ on $L$ which preserves $S_{F}$. This action of $\delta_{F}(\varphi)$ on $S_{F}$ is known as quantisation and is defined for functions $\varphi$ in $C_{F}^{1}$. Functions in $C_{F}^{0}$ quantise as zeroth order differential operators, that is as multiplication operators, and so can be considered to have been quantised in an already diagonal form. Since one is interested in the spectrum of the quantised operators it is clearly useful when one can choose $F$ so that $\varphi$ is in $C_{F}^{0}$.

In [7], [8] Kostant extends the above process to functions outside $C_{F}^{1}$, at least when there is a second polarisation transverse to $F$ and $F$ is real.

D. Simms [16], [17] carries out the above construction for the isotropic harmonic oscillator in $n$ dimensions using a polarisation $F$ for which the Hamiltonian $h$ is in $C_{F}^{0}$. However, $S_{F}$ in this case vanishes, there being no smooth solutions of the polarisation equations. Simms circumvents this difficulty by considering weak solutions. Such solutions form a space on which $h$ operates diagonally with spectrum given by the corrected Bohr-Sommerfeld condition [5] (this is due to the inclusion of $N_{F}^{1 / 2}$ in $L$ ) and with the same multiplicities as the usual quantum mechanical harmonic oscillator.

J. Sniatycki [18], [19] considers such weak polarised sections for general systems, showing that the support of the generalised sections is contained in the Bohr-Sommerfeld subset (that is the set of points $x$ where $h(x)$ is an energy satisfying the Bohr-Sommerfeld condition). Kostant has suggested an alternative approach which avoids using weak polarised sections. Namely, if one regards $S_{F}$ as $H^{0}\left(X ; \Im_{F}\right)$, then when this space vanishes one should examine the higher cohomology groups $H^{p}\left(X ; \delta_{F}\right)$. Since $\delta_{F}(\varphi)$ for $\varphi$ in $C_{F}^{1}$ acts on $\delta_{F}$, it also acts on each $H^{p}\left(X ; \delta_{F}\right)$, and we shall also regard this as quantisation.

It was quickly verified by Blattner, Simms, Sniatycki and the author that one certainly obtained the correct spectrum for the harmonic oscillator in one dimension on $H^{1}\left(X ; \varsigma_{F}\right)$. Sniatycki has considered these cohomology groups for real polarisations in [20] and [21]. In the present paper we shall treat the case of a general complex polarisation $F$ for which $C_{F}^{0}$ contains a function $h$ whose Hamiltonian flow is periodic. 
In $\$ 2$ we define the notion of prequantisation [6]. $\$ \S 3,4$ and 5 develop a calculus of differential forms defined on $F$ with values in $L$, that is, sections of $\wedge^{p} F^{*} \otimes L, F^{*}$ being the dual bundle of $F$. These forms give a resolution of $\delta_{F}$, due to Kostant, which gives a convenient realisation of $H^{p}\left(X ; \delta_{F}\right)$. If $h$ in $C_{F}^{0}$ generates a periodic one-parameter Hamiltonian flow $\sigma_{l}$, we let $l$ be the function obtained from parallel transport in $L$ around the (closed) orbits of $\sigma_{t}$. In $\S 6$ we define a map $J: \Gamma\left(\wedge^{p} F^{*} \otimes L\right) \rightarrow \Gamma\left(\wedge^{p-1} F^{*} \otimes L\right)$, such that if $\partial^{F}$ is the exterior derivative of these forms,

$$
J \circ \partial^{F}+\partial^{F} \circ J=1-l,
$$

the right-hand side meaning the operation of multiplication by the function $1-l$. We define the Bohr-Sommerfeld subset of $X$ to be all points $x$ with $l(x)=1$. If this subset is denoted by $Y$, we show $J$ defines a map $\int: H^{p}\left(X ; \delta_{F}\right)$ $\rightarrow H^{p-1}\left(Y ; \delta_{F \mid Y}\right)$, and under this map $(2 \pi i)^{-1} \delta_{F}(h)$ for $h$ in $C_{F}^{0}$, which is multiplication by $h$ on $H^{p}\left(X ; \delta_{F}\right)$, carries over to a diagonal operator on $H^{p-1}\left(Y ; \delta_{F \mid Y}\right)$. When the set $Z$ of orbits of $\sigma_{t}$ on $Y$ is a manifold, we show in $\S 7$ that we can further compose $\mathcal{J}$ with a map $\tau^{*-1}$ into $H^{p-1}\left(Z ; \delta_{\tilde{F}}\right)$, where $\tilde{F}$ is the quotient polarisation of $F$ and there is a line bundle $\tilde{L}$ over $Z$ induced by $L$. The composite map $H^{p}\left(X ; \delta_{F}\right) \rightarrow H^{p-1}\left(Z ; \delta_{F}\right)$ is shown to be an isomorphism for $p=1$ and injective for $p>1$. Finally, $\$ 8$ carries through these constructions for the isotropic harmonic oscillator in $n$ dimensions $(n>1)$ using Simms' polarisation. We show all the cohomology groups vanish except $H^{1}\left(X ; \delta_{F}\right) \cong H^{0}\left(Z ; \delta_{\tilde{F}}\right) . Y$ is, in this instance, a countable union of hypersurfaces $Y_{m}=h^{-1}(m+n / 2)$ with $m$ an integer. $Z_{m}$, the quotient of $Y_{m}$ by $\sigma_{t}$, is isomorphic to $P_{n-1}(\mathbf{C})$, and $\tilde{L} \mid Z_{m}$ is the $m$ th power of the positive generator of $H^{2}\left(P_{n-1}(\mathbf{C}) ; \mathbf{Z}\right)$ so the space $H^{0}\left(Z_{m} ; \delta_{\tilde{F}}\right)$ is a $\left(\begin{array}{c}n+m-1 \\ m\end{array}\right)$ dimensional complex vector space. Thus, on $H^{1}\left(X ; \mathfrak{S}_{F}\right), h$ operates with spectrum $m+n / 2$, with multiplicity $\left(\begin{array}{c}n+m-1 \\ m\end{array}\right)$, with $m$ a nonnegative integer. This agrees with Simms' results.

The author would like to thank R. Blattner, D. J. Simms, J. Sniatycki and, especially, B. Kostant for many useful conversations on quantisation. Thanks are also due to the Sonderforschungsbereich "Theoretische Mathematik", University of Bonn, for their hospitality and financial support during the period of this research.

2. Prequantisation. Let $(X, \omega)$ be a connected symplectic manifold. That is, $X$ is a smooth, connected, finite-dimensional manifold and $\omega$ a closed 2 -form which is nonsingular as a bilinear form on each tangent space. Denoting by $थ(X)$ the complex vector fields on $X$ and $\Omega^{p}(X)$ the complex $p$-forms, $\omega$ determines a linear isomorphism between $\Omega^{1}(X)$ and $2(X)$. The smooth functions $C(X)$ on $X$ coincide with $\Omega^{0}(X)$. As usual, denote by $d$ the exterior 
derivative $\Omega^{p}(X) \rightarrow \Omega^{p+1}(X)$, then to each function $\varphi \in C(X)$ is associated a 1-form $d \varphi \in \Omega^{1}(X)$ and, thus, a vector field $\xi_{\varphi} \in \mathcal{Q}(X)$ where

$$
\left.d \varphi=\xi_{\varphi}\right\lrcorner \omega .
$$

$\xi_{\varphi}$ is called the Hamiltonian vector field associated to $\varphi$.

Given two functions $\varphi, \psi \in C(X)$ their Poisson bracket is defined by

$$
[\varphi, \psi]=\xi_{\varphi} \psi=\omega\left(\xi_{\psi}, \xi_{\varphi}\right) .
$$

This bracket makes $C(X)$ a Lie algebra and the map $j: C(X) \rightarrow \mathcal{Q}(X)$ defined by $j(\varphi)=\xi_{\varphi}$ is a homomorphism of Lie algebras whose kernel consists of the constant functions on $X$.

Quantisation is the construction of representations of $C(X)$ with this Lie algebra structure, or of Lie subalgebras. The first part of the geometric quantisation procedure uses a Hermitian line bundle with connection $L^{\omega}$ over $X$, the curvature of the connection being $2 \pi i \omega$, that is satisfying

$$
\left[\nabla_{\xi}, \nabla_{\eta}\right] s-\nabla_{[\xi, \eta]} s=2 \pi i \omega(\xi, \eta) s
$$

for any section $s$ of $L^{\omega}$ and any pair $\xi, \eta$ of vector fields on $X$. This is only possible when $\omega$ is integral, which means $\omega$ has integral periods over integral homology cycles in $X$. In the case when this condition is satisfied, the set of isomorphism classes of such bundles can be identified with $H^{1}(X, T)$ ( $T$ is the group of complex numbers of modulus one) and the prequantisation of $C(X)$ is constructed on the space $\Gamma\left(L^{\omega}\right)$ of smooth sections of $L^{\omega}$ as follows. To each function $\varphi \in C(X)$ we associate a first order operator $\delta(\varphi): \Gamma\left(L^{\omega}\right) \rightarrow \Gamma\left(L^{\omega}\right)$ by setting

$$
\delta(\varphi)=\nabla_{\xi_{\varphi}}+2 \pi i \varphi
$$

By virtue of equation (1) $\delta$ satisfies

$$
\delta([\varphi, \psi])=\delta(\varphi) \delta(\psi)-\delta(\psi) \delta(\varphi)
$$

for $\varphi, \psi \in C(X)$, so $\delta$ is a homomorphism of Lie algebras, where the operators on $L^{\omega}$ are given their usual commutator bracket Lie algebra structure. Further details may be found in [1], [6], [8], [14], [15].

3. The Poincare lemma. A subtangent bundle on a smooth manifold $X$ is a smooth subbundle $F$ of the complexified tangent bundle $T X^{c}$. Let $\bar{F}$ be the conjugate of $F$ with respect to the real form $T X$ of $T X^{c}$ which is also a subtangent bundle. The codimension of $F$ will be $\operatorname{dim}_{C} T_{x} X^{c}-\operatorname{dim}_{\mathbf{C}} F_{x}$.

Denote by $U_{F}(U)$ the space of smooth sections of $F$ over the open set $U \subset X . F$ is said to be involutive if $\mathscr{U}_{F}(X)$ is a Lie subalgebra of $\mho_{(X)}$ 
$=\mathcal{Q}_{T X^{c}}(X)$. For any open set $U \subset X$ let $C_{F}(U)$ denote all the functions $\varphi$ on $U$ with $\xi(\varphi)=0$ for all $\xi$ in $\mathcal{Q}_{F}(U)$. The spaces $C_{F}(U)$ together with restriction maps form a presheaf; let $e_{F}$ denote the associated sheaf.

Let $F^{0} \subset T^{*} X^{c}$ be the subcotangent bundle of covectors which vanish on $F$. Thus $\varphi \in C_{F}(U)$ if and only if $d \varphi$ is a section of $F^{0}$ on $U$. If $F$ has codimension $m$ then $\wedge^{m} F^{0}=K^{F}$ is a complex line bundle over $X$ which we call the canonical bundle of $F$.

If there are functions $\varphi_{1}, \ldots, \varphi_{m}$ in $C_{F}(U)$ with $\left(d \varphi_{1}, \ldots, d \varphi_{m}\right)$ a frame of $F^{0}$ at each point of $U$, then $\left(\varphi_{1}, \ldots, \varphi_{m}\right)$ is called a $C_{F}$-coordinate system and $U$ a $C_{F}$-coordinate neighbourhood. $F$ is said to be integrable if $X$ can be covered by $C_{F}$-coordinate neighbourhoods. Note that if $F$ is integrable, it is automatically involutive. The converse is not, in general, true.

THEOREM 1 (FROBENIUS AND NIRENBERG). If $F$ is involutive and if either

(i) $\operatorname{dim}_{\mathrm{C}} F_{x} \cap \bar{F}_{x}$ is constant and $F+\bar{F}$ is involutive; or

(ii) $X$ is a real analytic manifold and $F$ an analytic subtangent bundle, then $F$ is integrable.

Proof. (i) This case is just the complex Frobenius theorem proved by Nirenberg [10] and Hörmander [4].

(ii) We reduce this to case (i) as follows. The result is local so we only need prove that a specific point $x$ in $X$ has a $C_{F}$-coordinate neighbourhood. Without loss of generality we may suppose $X$ is $\mathbf{R}^{n+m}, x$ is the origin and $F$ is given by $n$ analytic vector fields $\xi_{1}, \ldots, \xi_{n}$ which can be expressed in terms of coordinates $x_{1}, \ldots, x_{n+m}$ by

$$
\xi_{j}=\sum_{k=1}^{n+m} a_{k j}\left(x_{1}, \ldots, x_{n+m}\right) \frac{\partial}{\partial x_{k}}, \quad j=1, \ldots, n .
$$

Each $a_{k j}$ is an analytic function of $x_{1}, \ldots, x_{n+m}$, and so extends by analytic continuation to a holomorphic function $\hat{a}_{k j}$ on $\mathbf{C}^{m+n}$. That $F$ be involutive means there are analytic functions $c_{j k l}$ such that

$$
\left[\xi_{j}, \xi_{k}\right]=\sum_{l=1}^{n} c_{j k l} \xi_{l}, \quad j, k=1, \ldots, n .
$$

Let $\hat{c}_{j k l}$ be the analytic continuations to $\mathbf{C}^{m+n}$ of the $c_{j k l}$ and define holomorphic vector fields $\hat{\xi}_{j}$ on $\mathbf{C}^{m+n}$ by

$$
\hat{\xi}_{j}=\sum_{k=1}^{m+n} \hat{a}_{k j} \frac{\partial}{\partial z_{k}}, \quad j=1, \ldots, n ;
$$

then it follows that

$$
\left[\hat{\xi}_{j}, \hat{\xi}_{k}\right]=\sum_{l=1}^{n} \hat{c}_{j k l} \hat{\xi}_{l}, \quad j, k=1, \ldots, n
$$


and hence the distribution $\hat{F}$ on $\mathbf{C}^{m+n}$ spanned by $\hat{\xi}_{1}, \ldots, \hat{\xi}_{n}$ and $\partial / \partial \bar{z}_{1}, \ldots$, $\partial / \partial \bar{z}_{m+n}$ is smooth, involutive and of codimension $m$. Moreover $\hat{F}_{z} \cap \hat{F}_{z}$ has constant dimension $2 n$ and $\hat{F}+\hat{F}_{z}$ is involutive since it equals all of $\left(T \mathbf{C}^{m+n}\right)^{c}$. Thus part (i) implies the existence of a $C_{F^{-}}$coordinate system $\psi_{1}, \ldots, \psi_{m}$ on a neighbourhood of the origin in $\mathbf{C}^{m+n}$. The restrictions $\varphi_{1}, \ldots, \varphi_{m}$ to $\mathbf{R}^{m+n}$ give the required $C_{F^{-}}$-coordinate system on a neighbourhood of the origin.

The validity of the theorem in case (ii) may be useful in the case $X$ is a homogeneous space for a Lie group $G$ and $F$ is $G$-invariant, since in this case $X$ and $F$ are then analytic.

Denote by $\Omega_{F}^{p}(U)$ the sections of $\wedge^{p} F^{*}$ over an open set $U$ in $X . \Omega_{F}^{0}(U)$ is just $C(U)$ the space of smooth functions on $U$. If $F$ is involutive we can define a differential $d^{F}: \Omega_{F}^{p}(U) \rightarrow \Omega_{F}^{p+1}(U)$ in the usual way, namely regarding sections of $\wedge^{p} F^{*}$ as alternating $C(U)$-multilinear maps of $\chi_{F}(U)$ into $C(U)$, we define, for $\alpha \in \Omega_{F}^{p}(U)$,

$$
\begin{aligned}
\left(d^{F} \alpha\right)\left(\xi_{1}, \ldots, \xi_{p+1}\right)= & \sum_{i=1}^{p+1}(-1)^{i+1} \xi_{i}\left[\alpha\left(\xi_{1}, \ldots, \hat{\xi}_{i}, \ldots, \xi_{p+1}\right)\right] \\
& +\sum_{i<j} \alpha\left(\left[\xi_{i}, \xi_{j}\right], \xi_{1}, \ldots, \hat{\xi}_{i}, \ldots, \hat{\xi}_{j}, \ldots, \xi_{p+1}\right)
\end{aligned}
$$

where $\xi_{i} \in \mathcal{Q}_{F}(U)$ for $i=1, \ldots, p+1$. Then $\left(d^{F}\right)^{2}=0$ and $d^{F} \varphi=d \varphi \mid F$ for $\varphi \in C(X)$. In fact $C_{F}(U)=\left\{\varphi \in C(U) \mid d^{F} \varphi=0\right\}$. The differential complex

$$
\Omega_{F}^{0}(X) \stackrel{d^{F}}{\longrightarrow} \Omega_{F}^{1}(X) \stackrel{d^{F}}{\longrightarrow} \cdots \stackrel{d^{F}}{\longrightarrow} \Omega_{F}^{n}(X) \stackrel{d^{F}}{\longrightarrow} 0
$$

has cohomology groups which we denote by $H^{p}\left(\Omega_{F}^{*}(X)\right)$.

The sheaf $e_{F}$ also has cohomology groups $H^{p}\left(X ; \mathcal{C}_{F}\right)$ associated to it. Notice that

$$
H^{0}\left(X ; \bigodot_{F}\right)=\Gamma\left(\bigodot_{F}\right)=C_{F}(X)=\operatorname{Ker} d^{F}: C(X) \rightarrow \Omega_{F}^{1}(X)=H^{0}\left(\Omega_{F}^{*}(X)\right) .
$$

In fact we also have

THEOREM 2. If condition (i) of Theorem 1 holds for an involutive $F$ then $H^{p}\left(X ; \bigodot_{F}\right)$ is isomorphic to $H^{p}\left(\Omega_{F}^{*}(X)\right)$ for all $p$. More is true: if $Q_{F}^{p}$ denotes the sheaf of germs of local smooth sections of $\wedge^{p} F^{*}$ with induced maps $d^{F}: \mathbb{Q}_{F}^{p}$ $\rightarrow \mathbb{Q}_{F}^{p+1}$, and noting that $\mathfrak{C}_{F}=\operatorname{Ker} d^{F}: \mathbb{Q}_{F}^{0} \rightarrow \mathbb{Q}_{F}^{1}$, we have

$$
0 \rightarrow e_{F} \hookrightarrow \mathbb{Q}_{F}^{0} \stackrel{d^{F}}{\longrightarrow} \mathbb{Q}_{F}^{1} \stackrel{d^{F}}{\longrightarrow} \cdots \stackrel{d^{F}}{\longrightarrow} \mathbb{Q}_{F}^{n} \stackrel{d^{F}}{\longrightarrow} 0
$$

is a fine resolution of $\mathcal{C}_{F}$ giving rise to the above isomorphisms.

PRoof. The sheaves $Q_{F}^{p}$ are clearly fine, and since $\left(d^{F}\right)^{2}=0$, it is only necessary to prove a Poincare lemma for $d^{F}$. That is, we must show, given an 
open set $U$ in $X$ and a section $\alpha$ of $\wedge^{p} F^{*}, p \geqslant 1$, on $U$ with $d^{F} \alpha=0$, then any point $x$ in $U$ has a neighbourhood $V$ on which there is a section $\beta$ of $\wedge^{p-1} F^{*}$ and $d^{F} \beta=\alpha \mid V$. Without loss of generality we may suppose $U$ is a $C_{F}$-coordinate neighbourhood with $C_{F}$-coordinates $v_{1}, \ldots, v_{l}, z_{l+1}, \ldots, z_{m}$, where $z_{j}=x_{j}+i y_{j}$ and $d v_{1}, \ldots, d v_{l}, d x_{l+1}, \ldots, d x_{m}, d y_{l+1}, \ldots, d y_{m}$ are linearly independent at each point of $U$. We may add $k=n+l-m$ real functions $u_{1}, \ldots, u_{k}$ to obtain a coordinate system $u_{1}, \ldots, u_{k}, v_{1}, \ldots, v_{l}$, $x_{l+1}, \ldots, x_{m}, y_{l+1}, \ldots, y_{m}$ on $U . F$ is then spanned on $U$ by $\partial / \partial u_{1}, \ldots$, $\partial / \partial u_{k}, \partial / \partial \bar{z}_{l+1}, \ldots, \partial / \partial \bar{z}_{m}$. If $k=0$ Chern's proof [2] of the Dolbeault lemma adapts to this situation, whilst if $k=n$ the proof of the real Poincare lemma [23] will work. For $0<k<n$ it is necessary to combine the two proofs as follows:

Let $\varphi_{i}=v_{i}, i=1, \ldots, l$, and $\varphi_{i}=z_{i}, i=l+1, \ldots, m$, be the $C_{F}$-coordinates described above which will remain fixed throughout the argument. Also let $\psi_{i}=u_{i}, i=1, \ldots, k$, and $\psi_{i}=\bar{z}_{i+l-k}, i=k+1, \ldots, n$; then $d^{F} \psi_{1}, \ldots, d^{F} \psi_{n}$ give a basis for $F^{*}$ on $U$. We shall say an element $\alpha$ $\in \Omega_{F}^{p}(U)$ is $j$-dependent if the expansion $\alpha=\sum_{i_{1} \ldots i_{p}} a_{i_{1}}, \ldots, i_{p} d^{F} \psi_{i_{1}} \wedge \ldots$ $\wedge d^{F} \psi_{i_{p}}$ involves only $d^{F} \psi_{i}$ with $i \leqslant j$. We make the following hypothesis:

$\left(\mathrm{H}_{j}\right)$ If $\alpha$ is $j$-dependent on $U$ with $d^{F} \alpha=0$ and $x$ in $U$ there is a neighbourhood $V$ of $x$ in $U$ and a form $\beta$ on $V$ which is $j$-dependent with $d^{F} \beta=\alpha \mid V$.

We are trying to prove $\left(\mathrm{H}_{n}\right)$ whilst for nonzero $\alpha$ the least value which $j$ can take is the degree $p$ of $\alpha$. In this case $\alpha=f d^{F} \psi_{1} \wedge \cdots \wedge d^{F} \psi_{p}$ and $d^{F} \alpha=0$ if and only if $\xi_{j}(f)=0$ for all $j>p$ where $\xi_{1}, \ldots, \xi_{n}, \eta_{1}, \ldots, \eta_{m}$ is the dual frame field to $d \psi_{1}, \ldots, d \psi_{n}, d \varphi_{1}, \ldots, d \varphi_{m}$. If $j \leqslant k, \xi_{j}$ is $\partial / \partial u_{j}$ and if $j>k, \xi_{j}$ is $\partial / \partial \bar{z}_{j+l-k}$. In either case it is possible to find a function $g$ on a neighbourhood $V$ of $x$ with $\xi_{p}(g)=f$ and $\xi_{j}(g)=0, j>p$. Then $\alpha$ $=\xi_{p}(g) d^{F} \psi_{1} \wedge \cdots \wedge d^{F} \psi_{p}=d^{F}\left[(-1)^{p-1} g d^{F} \psi_{1} \wedge \cdots \wedge d^{F} \psi_{p-1}\right]$ on $V$ which proves $\left(\mathrm{H}_{p}\right)$.

To prove $\left(\mathrm{H}_{n}\right)$ we proceed by induction on $j$. That is, we assume $\left(\mathrm{H}_{j}\right)$ proven and suppose $\alpha$ is a $j+1$-dependent $p$-form on $U$ with $d^{F} \alpha=0$ and $x$ is in $U$. We can write $\alpha=\alpha_{1}+d^{F} \psi_{j+1} \wedge \alpha_{2}$ with $\alpha_{1}$ and $\alpha_{2}$ being $j$-dependent forms on $U$. It follows from $d^{F} \alpha=0$ that $\xi_{k}\left(\alpha_{2}\right)=0$ for $k>j+1$, where by $\xi_{k}\left(\alpha_{2}\right)$ we mean the form $\sum_{i_{1}} \cdots i_{p-1} \xi_{k}\left(a_{i_{1}}, \ldots, i_{p-1}\right) d^{F} \psi_{i_{1}} \wedge \cdots \wedge d^{F} \psi_{i_{p-1}}$ if $\alpha_{2}=\sum_{i_{1} \cdots i_{p-1}} a_{i_{1}} \cdots i_{p-1} d^{F} \psi_{i_{1}} \wedge \cdots i_{p-1} \wedge d^{F} \psi_{i_{p-1}}^{F_{1}}$. As above we can find a $j-$ dependent $p-1$-form $\beta_{1}$ on a neighbourhood $V_{1}$ of $x$ in $U$ with $\alpha_{2}=\xi_{j+1}\left(\beta_{1}\right)$ and $\xi_{k}\left(\beta_{1}\right)=0, k>j+1$. But then

$$
\begin{aligned}
d^{F} \beta_{1} & =\sum_{i=1}^{n} d^{F} \psi_{i} \wedge \xi_{i}\left(\beta_{1}\right)=\sum_{i=1}^{j} d^{F} \psi_{i} \wedge \xi_{i}\left(\beta_{1}\right)+d^{F} \psi_{j+1} \wedge \alpha_{2} \\
& =\beta_{2}+d^{F} \psi_{j+1} \wedge \alpha_{2}
\end{aligned}
$$


with $\beta_{2}$ a $j$-dependent $p$-form on $V_{1}$. Thus $\alpha=\alpha_{1}+d^{F} \beta_{1}-\beta_{2}$ and $\alpha_{1}-\beta_{2}$ is $j$-dependent. From $d^{F} \alpha=0$ follows $d^{F}\left(\alpha_{1}-\beta_{2}\right)=0$, and since we are assuming $\left(\mathrm{H}_{j}\right)$, it follows that $\alpha_{1}-\beta_{2}=d^{F} \beta_{3}$ on some neighbourhood $V$ of $x$ in $V_{1}$, and then if $\beta=\beta_{1}+\beta_{3}$, we have $d^{F} \beta=\alpha \mid V$. Thus $\left(\mathrm{H}_{j}\right)$ implies $\left(\mathrm{H}_{j+1}\right)$ and, hence, the desired result.

We use Theorem 2 to identify $H^{p}\left(X ; \bigodot_{F}\right)$ and $H^{p}\left(\Omega_{F}^{*}(X)\right)$.

CoRollary 1. Assume the same conditions as in Theorem 2. Let $\omega \in \Omega^{2}(X)$ be a given closed 2-form whose restriction to $F$ vanishes. Then each $x$ in $X$ has a neighbourhood $U$ with $a$ 1-form $\alpha_{0}$ such that (i) $\alpha_{0} \mid F=0$ and (ii) $d \alpha_{0}=\omega \mid U$.

Proof. Since $d \omega=0, x$ has a neighbourhood $U_{1}$ on which there is a l-form $\alpha_{1}$ with $d \alpha_{1}=\omega \mid U_{1}$. Let $\tilde{\alpha}_{1}$ be the restriction of $\alpha_{1}$ to $F$. Then $d^{F} \tilde{\alpha}_{1}=0$. Thus there is a neighbourhood $U$ of $x$ in $U_{1}$ and a function $\varphi$ on $U$ with $d^{F} \varphi=\tilde{\alpha}_{1} \mid U$. Let $\alpha_{0}=\alpha_{1} \mid U-d \varphi$. Then $d \alpha_{0}=\omega \mid U$ and $\alpha_{0}\left|F=\tilde{\alpha}_{1}\right| U$ $-d^{F} \varphi=0$.

REMARK 1. Theorem 2 does not remain true if instead we assume condition (ii) of Theorem 1. For suppose $X=\mathbf{R}^{3}$ and $F$ is spanned by the vector field

$$
P=\frac{1}{2}\left(\partial / \partial x_{1}+i \partial / \partial x_{2}\right)-i\left(x_{1}+i x_{2}\right) \partial / \partial x_{3} .
$$

Clearly $F$ is an analytic complex distribution. Lewy [9] shows there are $C^{\infty}$ functions $f$ on $X$ with the property that $P \varphi=f$ has no $C^{\infty}$ solution on any open set. Choose such a function $f$ and let $\beta$ be the section of $F^{*}$ defined by $\beta(P)=f$. Then $d^{F} \beta=0$, but there are no local solutions to $\beta=d^{F} \varphi$.

REMARK 2. If $F=T X^{c}$, Theorem 2 is the usual de Rham theorem since $e_{F}$ is the constant sheaf $\underline{C}$. If $T X^{c}$ is the internal direct sum $F \oplus \bar{F}$, then defining $J \in$ End $T X$ as $-i$ on $F$ and $i$ on $F, X$ becomes a complex manifold, $C_{F^{-}}$ coordinates are holomorphic coordinate systems and $e_{F}$ is the sheaf of germs of holomorphic functions. Theorem 2 in this case contains the $\bar{\partial}$ resolution of $e_{F}, \Omega_{F}^{p}(X)$ easily being identified with $\Omega^{0, p}(X)$ the forms of type $(0, p)$.

We shall say an involutive tangent subbundle is strongly integrable if it satisfies condition (i) of Theorem 1 .

EXAMPLE 1. Let $(X, \omega)$ be a symplectic manifold. A polarisation of $(X, \omega)$ is an involutive subtangent bundle $F$ of $T X^{c}$ with $\omega_{x}(\xi, \eta)=0$ for all $\xi, \eta \in F_{x}$, $x \in X$ and $\operatorname{dim}_{\mathrm{C}} F_{x}=\frac{1}{2} \operatorname{dim} X=n . F$ is said to be admissible if it is strongly integrable. The corollary to Theorem 2 implies the existence of a 1 -form $\alpha_{0}$ on some neighbourhood $U$ of any point with $\omega \mid U=d \alpha_{0}$ and $\alpha_{0} \in \Gamma\left(F^{0} \mid U\right) . F$ is said to be real if $F=\bar{F}$. Then $F=D^{c}$ is the complexification of a real integrable distribution $D$. Let $U_{1} \subset U$ be an open set on which there exist $C_{F^{-}}$ coordinates $q_{1}, \ldots, q_{n}$; then $d q_{1}, \ldots, d q_{n}$ form a frame at each point of $U_{1}$ for $F^{0}$, so there are functions $p_{1}, \ldots, p_{n}$ with $\alpha_{0} \mid U_{1}=\sum_{i=1}^{n} p_{i} d q_{i}$. We may assume the $p_{i}$ 's and $q_{i}^{\prime}$ 's are real; then $\omega\left|U_{1}=d \alpha_{0}\right| U_{1}=\sum_{i=1}^{n} d p_{i} \wedge d q_{i}$ 
implies $\left(p_{1}, \ldots, p_{n}, q_{1}, \ldots, q_{n}\right)$ form a Darboux coordinate system on $U_{1}$. Moreover $\left(\partial / \partial p_{1}, \ldots, \partial / \partial p_{n}\right)$ form a frame for $D$ at each point of $U_{1}$.

4. Line bundle valued forms. Let $\pi: L \rightarrow X$ be a line bundle over $X$ and $F$ a subtangent bundle. An $F$-connection in $L$ is a linear map

$$
\nabla: \Gamma(L) \rightarrow \Gamma\left(F^{*} \otimes L\right)
$$

such that for any section $s$ of $L$ and smooth function $\varphi$ on $X$,

$$
\nabla(\varphi s)=\varphi \nabla s+d^{F} \varphi \otimes s .
$$

For $\xi \in \mathcal{U}_{F}(X)$ we define $\nabla_{\xi} \in \operatorname{End} \Gamma(L)$ by $\nabla_{\xi} s=(\nabla s)(\xi)$ regarding $F^{*}$ $\otimes L$ as $\operatorname{Hom}(F, L)$. When $F$ is involutive the curvature of $\nabla$ is the form $\rho \in \Omega_{F}^{2}(X)$ such that

$$
\left[\nabla_{\xi}, \nabla_{\eta}\right] s-\nabla_{[\xi, \eta]} s=\rho(\xi, \eta) s
$$

for all $\xi, \eta$ in $\mathcal{Q}_{F}(X)$. We extend $\nabla$ to a map $\partial^{F}: S_{F}^{p}(L) \rightarrow S_{F}^{p+1}(L)$ where $S_{F}^{p}(L)=\Gamma\left(\wedge^{p} F^{*} \otimes L\right)\left(\right.$ so $\left.\nabla: S_{F}^{0}(L) \rightarrow S_{F}^{1}(L)\right)$ by defining

$$
\begin{aligned}
\left(\partial^{F} \alpha\right)\left(\xi_{1}, \ldots, \xi_{p+1}\right)= & \sum_{i=1}^{p+1}(-1)^{i+1} \nabla_{\xi_{i}}\left[\alpha\left(\xi_{1}, \ldots, \hat{\xi}_{i}, \ldots, \xi_{p+1}\right)\right] \\
& +\sum_{i<j}(-1)^{i+j} \alpha\left(\left[\xi_{i}, \xi_{j}\right], \xi_{1}, \ldots, \hat{\xi}_{i}, \ldots, \hat{\xi}_{j}, \ldots, \xi_{p+1}\right)
\end{aligned}
$$

where $\xi_{i} \in \mathcal{U}_{F}(X), i=1, \ldots, p+1$.

There is also an action of $\Omega_{F}^{p}(X)$ on $S_{F}^{q}(L)$ into $S_{F}^{p+q}(L)$, denoted by $\alpha \wedge \beta$, making $S_{F}^{*}(L)=\Sigma_{p \geqslant 0} S_{F}^{p}(L)$ into a left $\Omega_{F}^{*}(X)=\sum_{p \geqslant 0} \Omega_{F}^{p}(X)$-module. Then we have for any $\alpha \in S_{F}^{*}(L), \beta \in \Omega_{F}^{p}(X)$,

$$
\partial^{F}\left(\partial^{F} \alpha\right)=\rho \wedge \alpha ; \quad \partial^{F}(\beta \wedge \alpha)=\left(d^{F} \beta\right) \wedge \alpha+(-1)^{p} \beta \wedge \partial^{F} \alpha .
$$

Thus $\left(\partial^{F}\right)^{2}=0$ if $\rho=0$, in which case we say $\nabla$ is flat.

EXAmple 2. The canonical bundle $K=K^{F}=\wedge^{m} F^{0}$ of $F$ has a flat $F$ connection as follows: $\Gamma(K) \subset \Omega^{m}(X)$ so $d \alpha$ is an $m+1$-form on $X$ for $\alpha \in \Gamma(K)$. Let $\Omega_{F}^{m, p}(X)$ be all $m+p$-forms $\beta \in \Omega^{m+p}(X)$ with $\alpha \wedge \beta=0$ for all $\alpha$ in $\Gamma\left(F^{0}\right)$. Then $\Omega_{F}^{m, 0}(X)=\Gamma(K)$ and $d \Omega_{F}^{m, p}(X) \subset \Omega_{F}^{m, p+1}(X)$. There is a canonical isomorphism $\Omega_{F}^{m, p}(X) \stackrel{\sim}{\longrightarrow} \Gamma\left(\wedge^{p} F^{*} \otimes K\right)=S_{F}^{p}(K)$ defined by $\alpha \wedge \beta \rightarrow \alpha \mid F \otimes \beta$ for $\alpha$ in $\Omega^{p}(X), \beta$ in $\Gamma(K)$. Thus $\Omega_{F}^{m, 1}(X)$ is isomorphic to $S_{F}^{1}(K)$. Let $\nabla$ be the composition

$$
\Gamma(K) \stackrel{d}{\rightarrow} \Omega_{F}^{m, 1}(X) \cong S_{F}^{1}(K)=\Gamma\left(F^{*} \otimes K\right) .
$$

This is the required $F$-connection, which is flat since $d^{2}=0$. The differential $\partial^{F}: S_{F}^{p}(K) \rightarrow S_{F}^{p+1}(K)$ coincides with 


$$
S_{F}^{p}(K) \cong \Omega_{F}^{m, p}(X) \stackrel{d}{\rightarrow} \Omega_{F}^{m, p+1}(X) \cong S_{F}^{p+1}(K) .
$$

Returning to the general situation with $F$ an involutive tangent subbundle and $\nabla$ a flat $F$-connection in a line bundle $L$ over $X$, the spaces $S_{F \mid U}^{p}(L \mid U)$ form presheaves and maps of presheaves $\partial^{F}: S_{F \mid U}^{p}(L \mid U) \rightarrow S_{F \mid U}^{p+1}(L \mid U)$ which give rise to sheaves $\delta_{F}^{p}(L)$ and homomorphisms $\partial^{F}: \delta_{F}^{p}(L) \rightarrow \delta_{F}^{p+1}(L)$. Denote by $\delta_{F}$ the kernel of $\partial^{F}: \delta_{F}^{0}(L) \rightarrow \delta_{F}^{1}(L) . S_{F \mid U}^{0}(L \mid U)$ is the space of sections of $L$ over $U$ and, thus, $\delta_{F}^{0}(L)$ the sheaf of germs of sections of $L . \delta_{F}$ is the sheaf of germs of sections of $L$ which are covariant constant along $F$. We have thus a sequence

$$
0 \rightarrow \delta_{F} \hookrightarrow \delta_{F}^{0}(L) \stackrel{\partial^{F}}{\longrightarrow} \delta_{F}^{1}(L) \stackrel{\partial^{F}}{\longrightarrow} \cdots \stackrel{\partial^{F}}{\longrightarrow} \delta_{F}^{n}(L) \stackrel{\partial^{F}}{\longrightarrow} 0
$$

with $\partial^{F} \circ \partial^{F}=0$.

THEOREM 3. Let $F$ be an involutive, strongly integrable subtangent bundle on $X$ and $L$ a line bundle over $X$ with a flat $F$-connection $\nabla$; then the sequence (3) is a fine resolution of $\delta_{F}$.

Proof. This follows from the exactness of the sequence (2) which is guaranteed by Theorem 2. It is necessary only to show that if $\alpha \in S_{F \mid U}^{p}(L \mid U)$ satisfies $\partial^{F} \alpha=0$ and $x \in U$, then there is a neighbourhood $V$ of $x$ in $U$ and $\beta \in S_{F \mid V}^{p-1}(L \mid V)$ with $\partial^{F} \beta=\alpha \mid V$. Thus suppose $\alpha \in S_{F \mid U}^{p}(L \mid U)$ with $\partial^{F} \alpha$ $=0$ and $x \in U$. There is a neighbourhood $V_{1}$ of $x$ in $U$ with a nowhere vanishing section $s_{0}: V_{1} \rightarrow L$. Then one has $\alpha_{0} \in \Omega_{F}^{1}\left(V_{1}\right)$ defined by $\nabla s_{0}$ $=\alpha_{0} \otimes s_{0}$. Moreover the flatness of $\nabla$ implies $d^{F} \alpha_{0}=0$. Thus by Theorem 2 there is a neighbourhood $V_{2}$ of $x$ in $V_{1}$ and a function $\varphi_{0}$ on $V_{2}$ with $d^{F} \varphi_{0}=\alpha_{0} \mid V_{2}$. Set $s_{1}=e^{-\varphi_{0}} s_{0} \mid V_{2}$; then $\nabla s_{1}=0$ and $s_{1}$ vanishes nowhere on $V_{2}$. Thus there is $\alpha_{1}$ in $\Omega_{F}^{p}\left(V_{2}\right)$ with $\alpha \mid V_{2}=\alpha_{1} \otimes s_{1}$, and $\partial^{F} \alpha \mid V_{2}=\left(d^{F} \alpha_{1}\right) \otimes s_{1}$. $\partial^{F} \alpha=0$ implies $d^{F} \alpha_{1}=0$ and so there is a neighbourhood $V$ of $x$ in $V_{2}$ and $\beta_{1}$ in $\Omega_{F}^{p-1}(V)$ with $\alpha_{1} \mid V=d^{F} \beta_{1}$. Putting $\beta=\beta_{1} \otimes s_{1} \mid V, \partial^{F} \beta=d^{F} \beta_{1}$ $\otimes s_{1}\left|V=\left(\alpha_{1} \otimes s_{1}\right)\right| V=\alpha \mid V$, which proves the result.

COROLlaRY 2. Under the same conditions as in the theorem, for any $x$ in $X$ there is a neighbourhood $U$ of $x$ and a section $s_{1}: U \rightarrow L$ which vanishes nowhere and $\nabla s_{1}=0$ on $U$.

Proof. The section $s_{1}$ defined in the course of the proof of the theorem with $U=V_{2}$ satisfies these requirements.

The cohomology groups $H^{p}\left(X ; \delta_{F}\right)$ of the sheaf $\delta_{F}$ can now be identified with those of the complex

$$
S_{F}^{0}(L) \stackrel{\partial^{F}}{\longrightarrow} S_{F}^{n}(L) \stackrel{\partial^{F}}{\longrightarrow} \cdots \stackrel{\partial^{F}}{\longrightarrow} S_{F}^{n}(L) .
$$


One consequence of this is that these groups vanish in degrees higher than the fibre dimension of $F$.

Remark 3. Let $T X^{c}=F \oplus \bar{F}$ and give $X$ the complex structure as in Remark 2. A line bundle $\pi: L \rightarrow X$ is holomorphic if there is a covering $\left\{U_{i}\right\}$ of $X$ and nowhere vanishing sections $s_{i}: U_{i} \rightarrow L$ with $s_{j}=c_{i j} s_{i}$ on $U_{i} \cap U_{j}$ and $c_{i j} \in C_{F}\left(U_{i} \cap U_{j}\right)$. That is, the transition functions $c_{i j}$ are holomorphic. Then $L$ has a flat $F$-connection $\nabla$ defined by $\nabla s=d^{F} f_{i} \otimes s_{i}$ on $U_{i}$ if $s=f_{i} s_{i}$ on $U_{i}$. A section $s$ of $L$ is holomorphic if and only if $\nabla s=0 . \delta_{F}$ is the sheaf of germs of holomorphic sections of $L$ and (3) the $\bar{\partial}$ resolution of $\delta_{F}$.

EXAMPLE 3. Returning to the situation of Example 1, let $\pi: L \rightarrow X$ be a line bundle with flat $F$-connection $\nabla$ ( $L$ may be the line bundle with connection of prequantisation of $\S 2$ or such a line bundle tensored with a line bundle of $\frac{1}{2}$ forms normal to $F$ which has a canonical flat $F$-connection [1], [8], [15], [18]). $\delta_{F}$ is the sheaf of germs of polarised sections of $L . H^{0}\left(X ; \delta_{F}\right)$, denoted $S_{F}$ is the space of global polarised sections of $L$ and is the module on which quantisation [8] takes place. When $S_{F}$ vanishes, Kostant has suggested using the higher cohomology groups of $S_{F}$ for the quantisation process. (4) then gives a convenient representation of these cohomology groups in terms of $L$-valued forms defined on $F$. This example will be considered further in the later sections.

5. Lie derivatives. Let $F$ be a subtangent bundle on a manifold $X$. An infinitesimal automorphism of $F$ is a vectorfield $\xi$ such that $[\xi, \eta] \in \bigcup_{F}(X)$ for all $\eta \in \mathcal{U}_{F}(X)$. Then for any $\alpha \in \Omega_{F}^{p}(X)$ we define the Lie derivative $\theta(\xi) \alpha$ by

$$
(\theta(\xi) \alpha)\left(\xi_{1}, \ldots, \xi_{p}\right)=\xi\left[\alpha\left(\xi_{1}, \ldots, \xi_{p}\right)\right]-\sum_{i=1}^{p} \alpha\left(\xi_{1}, \ldots,\left[\xi, \xi_{i}\right], \ldots, \xi_{p}\right)
$$

for $\xi_{1}, \ldots, \xi_{p}$ in $\vartheta_{F}(X)$. Each $\xi$ in $\mho_{F}(X)$ is an infinitesimal automorphism of $F$ if $F$ is involutive; then defining $i(\xi): \Omega_{F}^{p}(X) \rightarrow \Omega_{F}^{p-1}(X)$ by

$$
(i(\xi) \alpha)\left(\xi_{1}, \ldots, \xi_{p-1}\right)=\alpha\left(\xi, \xi_{1}, \ldots, \xi_{p-1}\right)
$$

for $\xi_{1}, \ldots, \xi_{p-1}$ in $\vartheta_{F}(X)$ if $p \geqslant 1$ and $i(\xi) \varphi=0$ for $\varphi \in \Omega_{F}^{0}(X)$, then

$$
\theta(\xi)=d^{F} \circ i(\xi)+i(\xi) \circ d^{F}
$$

for $\xi \in \mathcal{U}_{F}(X)$.

If $\xi$ generates a one-parameter group $\sigma_{t}$ of diffeomorphisms, that is

$$
\xi \varphi=\left.(d / d t) \sigma_{-t}^{*} \varphi\right|_{t=0}, \quad \varphi \in C(X),
$$

then 


$$
\theta(\xi) \alpha=\left.(d / d t) \sigma_{-t}^{*} \alpha\right|_{t=0}, \quad \alpha \in \Omega_{F}^{*}(X),
$$

where $\sigma^{*} \alpha$ is defined for diffeomorphisms $\sigma$ with $\sigma_{*} F_{x}=F_{\sigma x}$ by

$$
\left(\sigma^{*} \alpha\right)_{x}\left(\xi_{1}, \ldots, \xi_{p}\right)=\alpha_{\sigma x}\left(\sigma_{*} \xi_{1}, \ldots, \sigma_{*} \xi_{p}\right)
$$

for $\alpha \in \Omega_{F}^{p}(X), \xi_{1}, \ldots, \xi_{p} \in F_{x}$.

Now let $\pi: L \rightarrow X$ be a line bundle with flat $F$-connection $\nabla$ and $F$ an involutive subtangent bundle. If $\xi \in \mathcal{Q}_{F}(X)$ and $\alpha \in S_{F}^{p}(L)$, define $\theta(\xi) \alpha$ $\in S_{F}^{p}(L)$ by

$$
(\theta(\xi) \alpha)\left(\xi_{1}, \ldots, \xi_{p}\right)=\nabla_{\xi}\left[\alpha\left(\xi_{1}, \ldots, \xi_{p}\right)\right]-\sum_{i=1}^{p} \alpha\left(\xi_{1}, \ldots,\left[\xi, \xi_{i}\right], \ldots, \xi_{p}\right)
$$

for $\xi_{1}, \ldots, \xi_{p}$ in $\vartheta_{F}(X)$. Then one has the identity

$$
\theta(\xi)=\partial^{F} \circ i(\xi)+i(\xi) \circ \partial^{F}
$$

where $i(\xi): S_{F}^{p}(L) \rightarrow S_{F}^{p-1}(L)$ is defined in the obvious way.

Suppose $\xi \in \mathcal{Q}_{F}(X)$ generates a one-parameter group $\sigma_{t}$; then to obtain an analogue of (5) we proceed as follows. Since $\xi \in \mathcal{U}_{F}(X)$ the curve $\gamma_{x}(t)$ $=\sigma_{-t} x$ through $x$ has tangent at each point in $F$ so that parallel transport along $\gamma_{x}$ in $L$ is defined with respect to $\nabla$. Let $P_{x, t}$ denote the transport map: $L_{\sigma_{t} x} \rightarrow L_{x}$ along $\gamma_{x}$ from $\sigma_{t} x$ to $x$. For $\alpha$ in $\Omega_{F}^{p}(X)$ define $\Sigma_{t} \alpha$ in $\Omega_{F}^{p}(X)$ by

$$
\left(\Sigma_{t} \alpha\right)_{x}\left(\xi_{1}, \ldots, \xi_{p}\right)=P_{x, t}\left[\alpha_{\sigma_{t} x}\left(\sigma_{t} x\left(\sigma_{t^{*}} \xi_{1}, \ldots, \sigma_{t^{*}} \xi_{p}\right)\right)\right]
$$

for $\xi_{1}, \ldots, \xi_{p}$ in $F_{x}$. Then we have

$$
\theta(\xi) \alpha=\left.(d / d t) \Sigma_{-t} \alpha\right|_{t=0},
$$

using for example the formula given in [6] for parallel transport in line bundles.

6. The periodic case. Continuing with the notation of $\$ 5$, suppose now $\sigma_{t}$ is a periodic one-parameter group so $\sigma_{t}=\sigma_{1+t}$ for all $t$ in $\mathbf{R}$. The lifting $\Sigma_{t}$ into $S_{F}^{p}(L)$, although it is a group, is no longer periodic in general. However $\Sigma_{1}$ is related to $\Sigma_{0}$ as follows: Since $\sigma_{1}=\sigma_{0}=\mathrm{id}_{X}$ the curve $\gamma_{x}$ is closed. Let $l(x)$ be the scalar in GL $(1, \mathrm{C})$ which equals $P_{x, 1}: L_{x} \rightarrow L_{x}$. Then for $\alpha$ in $S_{F}^{p}(L)$,

$$
\left(\Sigma_{1} \alpha\right)_{x}\left(\xi_{1}, \ldots, \xi_{p}\right)=P_{x, 1}\left[\alpha_{\sigma_{1} x}\left(\sigma_{1^{*}} \xi_{1}, \ldots, \sigma_{1^{*}} \xi_{p}\right)\right]=l(x) \alpha_{x}\left(\xi_{1}, \ldots, \xi_{p}\right)
$$

for $\xi_{i} \in F_{x}, i=1, \ldots, p$. So $\Sigma_{1} \alpha=l \alpha$. Thus

$$
\begin{aligned}
(l-1) \alpha & =\Sigma_{1} \alpha-\Sigma_{0} \alpha=\int_{0}^{1} \frac{d}{d s} \Sigma_{s} \alpha d s=\left.\int_{0}^{1} \frac{d}{d t} \Sigma_{s+t} \alpha\right|_{t=0} d s \\
& =\left.\int_{0}^{1} \Sigma_{s} \frac{d}{d t} \Sigma_{t} \alpha\right|_{t=0} d s=\int_{0}^{1} \Sigma_{s}[-\theta(\xi) \alpha] d s .
\end{aligned}
$$


Now one can easily check that $\Sigma_{s} \circ \theta(\xi)=\theta(\xi) \circ \Sigma_{s}$ and $\partial^{F} \circ \Sigma_{s}=\Sigma_{s}$ $\circ \partial^{F}$ so that

$$
(l-1) \alpha=-\theta(\xi) \int_{0}^{1} \Sigma_{s} \alpha d s
$$

and defining $I \alpha$ by $\int_{0}^{1} \Sigma_{s} \alpha d s$, we have

$$
(1-l) \alpha=\partial^{F} \circ i(\xi) \circ I \alpha+i(\xi) \circ I \circ \partial^{F} \alpha .
$$

If $J=i(\xi) \circ I$,

$$
J \circ \partial^{F} \alpha+\partial^{F} \circ J \alpha=(1-l) \alpha
$$

where the right-hand side of (7) is the operation of multiplying a form by the function $1-l$. This equation certainly makes sense for all $\alpha$ in $S_{F}^{p}(L)$ if $p \geqslant 1$, whilst for $p=0$ it becomes

$$
J \circ \partial F_{s}=(1-l) s, \quad s \in \Gamma(L) .
$$

Usually the bundles with which we deal have a parallel-transport invariant Hermitian structure so that the function $l$ takes its values in the unit circle $T$ (see [6]). We shall suppose this is so and that 1 is a regular value of $l$ as a map $l: X \rightarrow T$. Then $l^{-1}(1)=Y$ is a closed submanifold of $X$ of codimension one which we shall call the Bohr-Sommerfeld subset of the flow $\sigma_{t}$.

Proposition 1. Under the above assumptions $S_{F}=0$.

Proof. $S_{F}$ consists of all solutions of $\partial^{F} s=0$ with $s$ in $\Gamma(L)$. But if $\partial F_{s}=0$ we have $(1-l) s=J \circ \partial^{F}=0$ and so $s=0$ on $X-Y$. By continuity, since a codimension one manifold has dense complement, we have $s=0$ everywhere.

THEOREM 4. Again with the above assumptions, if $\pi: E \rightarrow X$ is a vector bundle, then a section (smooth) $s$ of $E$ vanishes on $Y$ if and only if there is a smooth section $r$ of $E$ with $s=(1-l) r$.

Proof. Clearly, if $s=(1-l) r$, then $s$ vanishes on $Y$. Suppose the converse has been proven for functions on $X$. We can cover $X$ by open sets $U$ on which there is a smooth frame $s_{1}, \ldots, s_{m}$ for $E$; then on $U, s=\sum_{i=1}^{m} f_{i} s_{i}$ for smooth functions $f_{1}, \ldots, f_{m}$. Moreover, $s$ vanishes on $U \cap Y$ if and only if all the functions $f_{i}$ do. Then there are functions $g_{i}$ with $f_{i}=(1-l) g_{i}$ and $s$ $=(1-l) \sum_{i=1}^{m} g_{i} s_{i}$. One can easily see that the local sections $\sum_{i=1}^{m} g_{i} s_{i}$ piece together to give a global section $r$ satisfying our requirements. It remains to prove the result for functions on sufficiently small open sets. 
Since 1 is a regular value of $l$ there are coordinates $x_{1}, \ldots, x_{N}$ on a convex open set $U$ in $X$ with $Y \cap U$ given by $x_{1}=0$ and $l=e^{i x_{1}}$ on $U$. Suppose $\varphi$ in $C(U)$ vanishes on $Y \cap U$. Now $\partial l / \partial x_{1}=i l$ is nonzero on $U$. The integral, defined for $y$ in $U$,

$$
\int_{0}^{1}\left(\frac{\partial l}{\partial x_{1}}\right)\left(t x_{1}(y), x_{2}(y), \ldots, x_{N}(y)\right) d t
$$

becomes $\partial l / \partial x_{1}(y)$ for $y$ in $Y \cap U$, which is nonzero. Thus by continuity, by shrinking $U$ if necessary, we can suppose the integral is nonzero throughout $U$. Then, if $\varphi$ vanishes on $U \cap Y$,

$$
\frac{\int_{0}^{1}\left(\partial \varphi / \partial x_{1}\right)\left(t x_{1}(y), x_{2}(y), \ldots, x_{N}(y)\right) d t}{\int_{0}^{1}\left(\partial l / \partial x_{1}\right)\left(t x_{1}(y), x_{2}(y), \ldots, x_{N}(y)\right) d t}
$$

defines a smooth function on $U$ which we denote by $-\psi$. For $y$ in $U-Y$, $x_{1}(y) \neq 0$ so

$$
\begin{aligned}
-\psi(y) & =\frac{\int_{0}^{x_{1}(y)}\left(\partial \varphi / \partial x_{1}\left(\left(t, x_{2}(y), \ldots, x_{N}(y)\right) d t\right.\right.}{\int_{0}^{x_{1}(y)}\left(\partial l / \partial x_{1}\right)\left(t, x_{2}(y), \ldots, x_{N}(y)\right) d t} \\
& =\varphi(y) /(l(y)-1) .
\end{aligned}
$$

Thus $\varphi=(1-l) \psi$ on $U-Y$ and since all the functions in this equation are continuous, it must continue to hold on all of $U$, proving the result.

Proposition 2. $l \in C_{F}(X)$.

Proof. For any function $\varphi$ and section $s$ of $L$ we have $\partial^{F}(\varphi s)=\varphi \partial^{F} s$ $+d^{F} \varphi \otimes s$. Applying this to (8) with $\varphi=1-l$ we have

$$
(1-l) \partial^{F} s-d^{F} l \otimes s=\partial^{F} \circ J \circ \partial^{F} .
$$

Now applying (7) to $\partial^{F} s$ we have

$$
(1-l) \partial^{F_{s}}=\left(J \circ \partial^{F}+\partial^{F} \circ J\right) \partial^{F_{s}}=\partial^{F} \circ J \circ \partial^{F_{s}} .
$$

Thus $d^{F} l \otimes s=0$. Since we can choose $s$ to be nonzero in a neighbourhood of any point we must have $d^{F} l=0$ or $l \in C_{F}(X)$.

COROLLARY 3. If $\alpha=(1-l) \beta$ for $\alpha$ and $\beta$ in $S_{F}^{p}(L)$ then

$$
\partial^{F} \alpha=(1-l) \partial^{F} \beta \text {. }
$$


COROllary 4. $F$ is tangent to $Y$ so $F \mid Y$ is a subtangent bundle on $Y$ and all the definitions make sense with $X$ replaced by $Y$.

Define a map $\delta: H^{p}\left(X ; \delta_{F}\right) \rightarrow H^{p-1}\left(Y ; \delta_{F \mid Y}\right)$ by $\hat{J}[\alpha]=[J \alpha \mid Y] . \hat{J}$ is well defined, since restricting to $Y$ commutes with the coboundary: for $\alpha$ in $S_{F}^{p}(L),\left(\partial^{F} \alpha\right)\left|Y=\partial^{F \mid Y} \alpha\right| Y$, and then (7) implies $\left(J \partial^{F} \alpha\right) \mid Y+\partial^{F \mid Y}(J \alpha \mid Y)=0$ since $l=1$ on $Y$.

THEOREM 5. $\hat{J}$ is an isomorphism for $p=1$ and a monomorphism for $p \geqslant 2$.

Proof. First the case $p=1$. $j$ maps $H^{1}\left(X ; \delta_{F}\right)$ into $S_{F \mid Y}$. Suppose $\hat{J}[\alpha]$ $=0$, which means $J \alpha$ is a section of $L$ with $\partial^{F} \alpha=0$ and $J \alpha$ vanishes on $Y$. By Theorem 4 there is a section $s$ of $L$ with $J \alpha=(1-l) s$ and so $\partial^{F} J \alpha$ $=(1-l) \partial^{F} s$. By (7) we then have

$$
(1-l) \partial^{F_{s}}=(1-l) \alpha-J \partial^{F} \alpha=(1-l) \alpha
$$

and so $\alpha=\partial^{F}$ showing $[\alpha]=0$ and $\mathcal{J}$ is injective. Now suppose $s$ in $S_{F \mid Y}$. Choose any section $\tilde{s}$ of $L$ which coincides with $s$ on $Y$. This we may do since $Y$ is a closed submanifold in $X$. Then $\left(\partial^{F} \tilde{s}\right)\left|Y=\partial^{F \mid Y} \tilde{s}\right| Y=\partial^{F \mid Y}{ }_{s}=0$ so $\partial^{F} \tilde{s}$ vanishes on $Y$ and, hence, there is a form $\alpha$ in $S_{F}^{1}(L)$ with $\partial^{F} \tilde{s}=(1-l) \alpha$. Clearly $\partial^{F} \alpha=0$. Also $J(1-l) \alpha=(1-l) J \alpha$ since $l \in C_{F}(X)$, and so $(1-l) J \alpha=J \partial F_{\tilde{s}}=(1-l) \tilde{s}$. Thus $\tilde{s}=J \alpha$ and, hence, $f[\alpha]=s$ showing $\delta$ is onto in degree one.

For $p \geqslant 2$ suppose $\hat{J}[\alpha]=0$. That is $J \alpha \mid Y=\partial^{F \mid Y} \beta$ for some $\beta$ in $S_{F \mid Y}^{p-2}(L \mid Y)$. Extend $\beta$ to an element $\tilde{\beta}$ in $S_{F}^{p-2}(L)$; then $J \alpha-\partial^{F} \tilde{\beta}$ varishes on $Y$ so there is $\gamma$ in $S_{F}^{p-1}(L)$ with $J \alpha-\partial^{F} \tilde{\beta}=(1-l) \gamma$. Then

$$
\partial^{F} J \alpha=(1-l) \partial^{F} \gamma
$$

and, since $\partial^{F} \alpha=0$, we have $(1-l) \alpha=(1-l) \partial^{F} \gamma$ or $\alpha=\partial^{F} \gamma$, showing $[\alpha]=0$ and $\hat{J}$ is a monomorphism.

REMARK 4. Often in applications $l$ has the form $\exp 2 \pi i h$ for a function $h$ on $X$; then $Y$ is the union $\cup_{m \in \mathbf{Z}} Y_{m}$ with $Y_{m}=h^{-1}(m) . h$ is in $C_{F}$ and so operates by multiplication on both $H^{p}\left(X ; \delta_{F}\right)$ and $H^{p-1}\left(Y ; \delta_{F \mid Y}\right)$ commuting with $\hat{J}$. Clearly

$$
H^{p-1}\left(Y ; \delta_{F \mid Y}\right)=\prod_{m \in \mathbf{Z}} H^{p-1}\left(Y_{m} ; \delta_{F \mid Y_{m}}\right)
$$

and $h$ has eigenvalues $m$ with eigenspaces $H^{p-1}\left(Y_{m} ; \delta_{F \mid Y_{m}}\right)$ when nonzero.

7. Passing to the quotient. Let $Y$ be a manifold, $\tau: Y \rightarrow Z$ a submersion of $Y$ onto $Z$. Suppose $\tilde{F}$ is a strongly integrable subtangent bundle on $Z$; then $F=\tau_{*}^{-1} \tilde{F}$ is a strongly integrable subtangent bundle on $Y$. In this case we say $F$ and $\tilde{F}$ are $\tau$-related. Denote by $V_{\tau}$ all tangents $\xi$ to $Y$ with $\tau_{*} \xi=0 . V_{\tau}$ is the 
bundle of vertical tangents and $V_{\tau} \subset F$. A vector field $\xi$ on $Y$ will be called vertical if $\xi_{x}$ is in $V_{\tau, x}$ for all $x$ in $Y$.

If $F$ and $\tilde{F}$ are $\tau$-related we have a map $\tau^{*}: \Omega_{\tilde{F}}^{p}(Z) \rightarrow \Omega_{F}^{p}(Y)$ for each $p \geqslant 0$ defined by

$$
\left(\tau^{*} \alpha\right)_{x}\left(\xi_{1}, \ldots, \xi_{p}\right)=\alpha_{\tau x}\left(\tau_{*} \xi_{1}, \ldots, \tau_{*} \xi_{p}\right)
$$

for all $\xi_{1}, \ldots, \xi_{p}$ in $F_{x}, x$ in $Y$ where $\alpha \in \Omega_{\tilde{F}}^{p}(Z) . \tau^{*}$ is a homomorphism of exterior algebras and intertwines $d^{F}$ and $d^{\tilde{F}}$. Moreover if $\xi$ in $\mathcal{Q}_{F}(Y)$ is $\tau$ related to $\eta$ in $\mho_{\tilde{F}}(Z)$, then $i(\xi) \circ \tau^{*}=\tau^{*} \circ i(\eta)$ and $\theta(\xi) \circ \tau^{*}=\tau^{*} \circ \theta(\eta)$. In particular, since a vertical vector field is $\tau$-related to zero, $i(\xi) \tau^{*} \alpha=0$, $\theta(\xi) \tau^{*} \alpha=0$ for all vertical $\xi$, and all $\alpha$ in $\Omega_{\tilde{F}}^{p}(Z)$. In fact one may easily prove

Proposition 3. $\beta$ in $\Omega_{F}^{p}(Y)$ is of the form $\beta=\tau^{*} \alpha$ for some $\alpha$ in $\Omega_{\tilde{F}}^{p}(Z)$ if and only if $i(\xi) \beta=0, \theta(\xi) \beta=0$ for all vertical $\xi$.

We shall now extend these results to line bundle valued forms. Suppose $\tau: Y \rightarrow Z$ is a submersion with connected fibres and $F$ and $\tilde{F}$ are $\tau$-related, strongly integrable subtangent bundles on $Y$ and $Z$ respectively. Let $\pi: L \rightarrow Y$ be a line bundle with a flat $F$-connection $\nabla$; then a submanifold $M \subset Y$ is said to be absolutely parallel if $T M \subset F \mid M$, and parallel transport along curves in $M$ depends only on the endpoints and not the path taken. Equivalently, the restriction of $L$ to $M$ with the induced connection (which is an ordinary connection since the whole tangent space $T M$ is in $F$ ) has trivial holonomy groups at each point. We shall assume that each fibre of $\tau$ is an absolutely parallel submanifold of $Y$.

Define an equivalence relation $\sim$ on $L$ by $q_{1} \sim q_{2}$ if and only if there is a curve $\gamma$ in $Y$ with $\tau \circ \gamma$ constant and parallel transport along $\gamma$ takes $q_{1}$ to $q_{2}$. Let $\tilde{L}$ denote the set of equivalence classes and $[q]$ the class containing $q$. Observing that $\tau(\pi(q))$ depends only on $[q]$, we define $\tilde{\tau}: \tilde{L} \rightarrow Z$ by $\tilde{\tau}[q]$ $=\tau(\pi(q))$. To show $\tilde{L}$ is a line bundle over $Z$ with a flat $\tilde{F}$-connection $\tilde{\nabla}$ induced by $\nabla$, we need the following basic lemma.

LEMMA 1. Let $\tau: Y \rightarrow Z$ be a submersion of $Y$ onto $Z$ with connected fibres, $F$ and $\tilde{F}$-related strongly integrable subtangent bundles and $\pi: L \rightarrow Y$ a line bundle with flat $F$-connection $\nabla$ such that the fibres of $\tau$ are absolutely parallel; then given $z$ in $Z$ there is a neighbourhood $V$ of and a section s: $\tau^{-1}(V) \rightarrow L$ which vanishes nowhere with $\nabla s=0$.

Proof. Take $x_{0}$ in $Y$ with $\tau\left(x_{0}\right)=z$. By Corollary 2 there is a neighbourhood $U_{0}$ of $x_{0}$ with a section $s_{0}: U_{0} \rightarrow L$ vanishing nowhere with $\nabla s_{0}=0$. Let $V=\tau\left(U_{0}\right)$. Extend $s_{0}$ to a section $s$ on all of $\tau^{-1}(V)$ as follows. Given $x$ in $\tau^{-1}(V)$, the connectedness of the fibres of $\tau$ implies the existence of a curve $\gamma$ from a point of $U_{0}$ to $x$ with $\tau \circ \gamma$ constant. We define $s(x)$ as the parallel 
transport of $s_{0}(\gamma(0))$ along $\gamma . s(x)$ is independent of the curve $\gamma$ chosen since the fibres of $\tau$ are absolutely parallel. $s$ vanishes nowhere since parallel transport is a linear isomorphism and $s_{0}$ vanishes nowhere. We must show $s$ is smooth and $\nabla s=0$.

Suppose $\gamma$ lies wholly in an open set $U_{1} \subset \tau^{-1}(V)$ with a nowhere vanishing section $s_{1}: U_{1} \rightarrow L$ with $\nabla s_{1}=0$. Then $U_{1} \cap U_{0}$ contains $\gamma(0)$ so is open and nonempty. Replacing $U_{1}$ by $U_{1} \cap \tau^{-1}\left(\tau\left(U_{1} \cap U_{0}\right)\right)$ if necessary, we can ensure also $U_{1} \subset \tau^{-1}\left(\tau\left(U_{1} \cap U_{0}\right)\right)$. $s \mid U_{1}=f s_{1}$ for some function (not yet proven to be smooth) $f$ on $U_{1}$. Since $s\left|U_{1} \cap U_{0}=s_{0}\right| U_{1} \cap U_{0}, f$ is smooth on $U_{1} \cap U_{0}$. Moreover, $\nabla s=\nabla s_{0}=0$ and $\nabla s_{1}=0$ on $U_{1} \cap U_{0}$ so $d^{F} f=0$ on $U_{1} \cap U_{0}$. But then $f=\tilde{f} \circ \tau$ on $U_{1} \cap U_{0}$ with $\tilde{f}$ a smooth function on $\tau\left(U_{1} \cap U_{0}\right)$ with $d^{\tilde{F}} \tilde{f}=0$. Consider $s^{\prime}=\tilde{f} \circ \tau \cdot s_{1}$ on $U_{1}$. It satisfies $\nabla s^{\prime}=0$ and is smooth. Since $\nabla s^{\prime}=0$, the value of $s^{\prime}$ at any point is obtained from the value at some point of $U_{1} \cap U_{0}$ by parallel transport in the fibres of $\tau$. But

$$
s^{\prime}\left|U_{1} \cap U_{0}=s\right| U_{1} \cap U_{0} .
$$

Then by uniqueness of parallel transport we must have $s^{\prime}=s \mid U_{1}$. Thus proving $s \mid U_{1}$ is smooth and $\nabla s \mid U_{1}=0$.

Now let $x$ in $\tau^{-1}(V)$ be arbitrary and $\gamma$ a curve from a point of $U_{0}$ to $x$ with $\tau \circ \gamma$ constant. By the compactness of the range of $\gamma$ and Corollary 2 it can be covered by finitely many open sets $U_{1}, \ldots, U_{n}$ with nowhere vanishing sections $s_{i}: U_{i} \rightarrow L$ with $\nabla s_{i}=0$ and $U_{i} \subset \tau^{-1}\left(\tau\left(U_{i} \cap U_{0}\right)\right)$. Replacing $U_{0}$ in the argument of the previous paragraph by $U_{0} \cup U_{1} \cup \cdots \cup U_{j}$, if $s$ is proved smooth on this set with $\nabla s=0$ there, then the same is true on $U_{0} \cup U_{1} \cup \cdots \cup U_{j+1}$. By induction $s$ is smooth on $U_{n}$ and $\nabla s=0$ there. Since $x$ was arbitrary we have shown $s$ is smooth on $\tau^{-1}(V)$ with $\nabla s=0$.

Given a (set theoretic) section $\tilde{s}$ of $\tilde{L}$ we define a section $\tau^{*} \tilde{s}$ of $L$ by letting $\left(\tau^{*} \tilde{s}\right)(x)$ be the unique element of $L_{x}$ with $\left[\left(\tau^{*} \tilde{s}\right)(x)\right]=\tilde{s}(\tau(x))$. It is clear that if $\nabla s=0$ on $\tau^{-1}(V)$ with $V$ open in $Z$ then $s=\tau^{*} \tilde{s}$ for a uniquely defined section $\tilde{s}$ of $\tilde{L}$. Suppose $(V, \psi)$ is a chart on $Z$; then $\left(\tilde{\pi}^{-1}(V), \varphi\right)$ defined by $\varphi(c \tilde{s}(z))=(\psi(z), \operatorname{Re} c, \operatorname{Im} c)$ is a chart on $Z$ and the collection of such charts defines an atlas for $\tilde{L}$ giving it the structure of a smooth line bundle. The sections $\tilde{s}: V \rightarrow \tilde{L}$ such that $\tau^{*} \tilde{s}$ is a smooth nowhere vanishing section of $L$ on $\tau^{-1}(V)$ with $\nabla \tau^{*} \tilde{s}=0$ (which exist because of Lemma 1) give a trivialisation of $\tilde{L}$. We define a flat $\tilde{F}$-connection consistently in $\tilde{L}$ by setting $\tilde{\nabla} \tilde{s}=0$ for such a section. Then if $\xi$ in $\mho_{F}(V)$ is $\tau$-related to $\eta$ in $\mho_{\tilde{F}}(Z)$ we have $\nabla_{\xi} \tau^{*} \tilde{s}=\tau^{*} \nabla_{\eta} \tilde{s}$ for any smooth section $\tilde{s}$ of $\tilde{L}$ and $s$ in $\Gamma(L)$ is of the form $\tau^{*} \tilde{s}$ for $\tilde{s}$ in $\Gamma(\tilde{L})$ if and only if $\nabla_{\xi} s=0$ for all vertical fields $\xi$.

Define $\tau^{*}: S_{\tilde{F}}^{p}(\tilde{L}) \rightarrow S_{F}^{p}(L)$ by

$$
\left(\tau^{*} \alpha\right)\left(\xi_{1}, \ldots, \xi_{p}\right)=\tau^{*}\left(\alpha\left(\eta_{1}, \ldots, \eta_{p}\right)\right)
$$


for all $\alpha$ in $S_{\tilde{F}}^{p}(L), \xi_{1}, \ldots, \xi_{p}$ in $\vartheta_{F}(Y), \eta_{1}, \ldots, \eta_{p}$ in ${ }^{2} \tilde{F}_{F}(Z)$ with $\xi_{i} \tau$-related to $\eta_{i}, i=1, \ldots, p$. Then we have $\partial^{F} \circ \tau^{*}=\tau^{*} \circ \partial^{F}, i(\xi) \circ \tau^{*}=\tau^{*} \circ i(\eta)$ and $\theta(\xi) \circ \tau^{*}=\tau^{*} \circ \theta(\eta)$ for $\xi$ in $\chi_{F}(Y), \eta$ in $\bigcup_{F}(Z)$ with $\xi \tau$-related to $\eta$. Let $\tilde{S}_{F}^{p}(L)=\tau^{*} S_{F}^{p}(\tilde{L})$. Then generalising Proposition 3 we have

Proposition 4. An element $\alpha$ of $S_{F}^{p}(L)$ lies in $\tilde{S}_{F}^{p}(L)$ if and only if $i(\xi) \alpha=0, \theta(\xi) \alpha=0$ for all vertical vector fields $\xi$.

$\tilde{S}_{F}^{p}(L)$ is stable under $\partial^{F}$, and the corresponding cohomology groups we denote by $\tilde{H}^{p}\left(Y ; \delta_{F}\right) . \tau^{*}$ defines an isomorphism of $H^{p}\left(Z ; \delta_{\tilde{F}}\right)$ with $\tilde{H}^{p}\left(Y ; \delta_{F}\right)$ for each $p$ and there is a natural map of $\tilde{H}^{p}\left(Y ; \delta_{F}\right)$ into $H^{p}\left(Y ; \delta_{F}\right)$ which need not be one-one nor onto.

Now we return to the situation of the previous section where $\sigma_{t}$ is a oneparameter group on a manifold $X$ which is periodic and generated by $\xi$ in $\mathcal{Q}_{F}(X)$ for $F$ a strongly integrable subtangent bundle. Also $\pi: L \rightarrow X$ is a line bundle with flat $F$-connection $\nabla$ and the parallel transport function $l$ around orbits of $\sigma_{t}$ is $T$-valued with 1 a regular value. $Y=l^{-1}(1)$ consists of all orbits which are absolutely parallel submanifolds. We shall suppose the set $Z$ of orbits of $\sigma_{t}$ on $Y$ is a smooth manifold with natural projection $\tau: Y \rightarrow Z$.

Theorem 6. If $\alpha$ is in $S_{F}^{p}(L)$ with $\partial^{F} \alpha=0$ then $J \alpha \mid Y$ is in $\tilde{S}_{F \mid Y}^{p}(L \mid Y)$ and $\tau^{*-1} \circ \mathcal{S}: H^{p}\left(X ; \delta_{F}\right) \rightarrow H^{p-1}\left(Z ; \delta_{F}\right)$ is an isomorphism for $p=1$ and injective for $p>1$.

Proof. $J \alpha=i(\xi) I \alpha$ and $\xi$ spans the vertical tangent space at each point. Since $\partial^{F \mid Y} J \alpha \mid Y=0$, it immediately follows that $J \alpha \mid Y$ is in $\tilde{S}_{F \mid Y}^{p}(L \mid Y)$ and, since $\partial^{F \mid Y} J \alpha \mid Y=0$, it defines a class $[J \alpha \mid Y]=\hat{J}[\alpha]$ in $\tilde{H}^{p}\left(Y ; \delta_{F \mid Y}\right)$. Then $\tau^{*-1} \circ \hat{J}[\alpha]$ is in $H^{p-1}\left(Z ; \delta_{\tilde{F}}\right)$ where $\tilde{F}$ is the quotient of $F$ by $\sigma_{t}$ which exists since $\xi$ is in $\mathcal{U}_{F}(X)$ and $F$ is invariant under $\sigma_{t}$. The remainder of the theorem follows from Theorem 5 .

8. The $n$-dimensional harmonic oscillator $(n>1)$. In this example we study the quantisation of the $n$-dimensional harmonic oscillator using a polarisation considered by Simms [16], [17]. We shall compute the cohomology groups $H^{p}\left(X ; \delta_{F}\right)$ of this polarisation, showing that they vanish for $p \neq 1$, whilst on $H^{1}\left(X ; \delta_{F}\right)$ the Hamiltonian acts with the correct spectrum and multiplicities.

The phase space of this example is $X=\mathbf{C}^{n}-\{0\}$, and we let $z_{1}, \ldots, z_{n}$ be the usual linear coordinates. The symplectic form $\omega$ is $(2 i)^{-1} \sum_{j=1}^{n} d z_{j} \wedge d \bar{z}_{j}$ $=d \alpha_{0}$ with $\alpha_{0}=-(2 i)^{-1} \sum_{j=1}^{n} \bar{z}_{j} d z_{j}$. As Hamiltonian we take $h$ $=\pi \sum_{j=1}^{n}\left|z_{j}\right|^{2}$ with corresponding Hamiltonian vector field $\xi_{h}$ $=2 \pi i \sum_{j=1}^{n}\left(z_{j} \partial_{j}-\bar{z}_{j} \bar{\partial}_{j}\right)$. We use the notation $\partial_{j}$ for $\partial / \partial z_{j}$ and $\bar{\partial}_{j}$ for $\partial / \partial \bar{z}_{j}$. The flow generated by $\xi_{h}$ is $\sigma_{t}\left(z_{1}, \ldots, z_{n}\right)=\left(e^{-2 \pi i t} z_{1}, \ldots, e^{-2 \pi i t} z_{n}\right)$ which has period one. $\alpha_{0}$ and $\omega$ are invariant under $\sigma_{t}$, and $\alpha_{0}\left(\xi_{h}\right)=-h$.

Let $\xi_{i j}=z_{i} \bar{\partial}_{j}-z_{j} \bar{\partial}_{i}$; then at any point, precisely $n-1$ of these vector fields 
are linearly independent and $\xi_{i j}(h)=0$ for all $i$ and $j$. If $U_{k}$ denotes the set $\left\{\left(z_{1}, \ldots, z_{n}\right) \in X \mid z_{k} \neq 0\right\}$, then $\xi_{i j}=z_{i} / z_{k} \xi_{k j}-z_{j} / z_{k} \xi_{k i}$. Thus $\xi_{k, 1}, \ldots$, $\xi_{k, k}, \ldots, \xi_{k, n}$ are linearly independent at each point of $U_{k}$. Let $F_{1}$ be the $(n-1)$-dimensional complex subtangent bundle spanned on $U_{k}$ by $\xi_{k, 1}, \ldots$, $\widetilde{\xi_{k, k}}, \ldots, \xi_{k, n}$; then since $\left[\xi_{i j}, \xi_{r, s}\right]=0$ for all $i, j, r, s, F_{1}$ is involutive. Also $\left[\xi_{h}, \xi_{r s}\right]=2 \pi i \xi_{r s}$ so that $F=F_{1} \oplus \mathbf{C} \xi_{h}$ is an $n$-dimensional involutive complex subtangent bundle, which is in fact a strongly integrable polarisation of $(X, \omega)$. Moreover, $h$ is in $C_{F}(X)$.

Let $\lambda$ be the section of $F^{*}$ which is 1 on $\xi_{h}$ and vanishes on $F_{l}$; then $\alpha_{0} \mid F=-h \cdot \lambda$. Consider the $n$-form

$$
\mu=\sum_{j=1}^{n} z_{j} d z_{1} \wedge \cdots \wedge d z_{j-1} \wedge d h \wedge d z_{j+1} \wedge \cdots \wedge d z_{n}
$$

Define $\zeta_{\alpha}^{k}$ on $U_{k}$ as $z_{\alpha} / z_{k}$; then $h, \zeta_{1}^{k}, \ldots, \widehat{\zeta_{k}^{k}}, \ldots, \zeta_{n}^{k}$ give $C_{F}$-coordinates on $U_{k}$ and

$$
\mu \mid U_{k}=z_{k}^{n} d \zeta_{1}^{k} \wedge \cdots \wedge d \zeta_{k-1}^{k} \wedge d h \wedge d \zeta_{k+1}^{k} \wedge \cdots \wedge d \zeta_{n}^{k}
$$

so that $\mu$ is a global nowhere vanishing section of $K^{F}=\wedge^{n} F^{0}$. Further $d \mu\left|U_{k}=n d z_{k} / z_{k} \wedge \mu\right| U_{k}$ showing $\nabla \mu\left|U_{k}=n z_{k}^{-1} d^{F} z_{k} \otimes \mu\right| U_{k}$. But $d^{F} z_{k}\left(\xi_{i j}\right)$ $=0$ whilst $d^{F} z_{k}\left(\xi_{h}\right)=\xi_{h}\left(z_{k}\right)=2 \pi i z_{k}$ which implies $d^{F} z_{k}=2 \pi i z_{k} \lambda \mid U_{k}$ and, hence, $\nabla \mu=2 \pi i n \lambda \otimes \mu$.

Let $N_{F}^{1 / 2}$ be the bundle of $\frac{1}{2}$-forms normal to $F$. (See [1], [8], [15], [18].) The only properties of $N_{F}^{1 / 2}$ needed here are that $N_{F}^{1 / 2} \otimes N_{F}^{1 / 2}$ is isomorphic to $K^{F}$ and $N_{F}^{1 / 2}$ has a flat $F$-connection $\nabla^{1 / 2}$ such that $\nabla^{1 / 2} \otimes 1+1 \otimes \nabla^{1 / 2}$ carries over into $\nabla$ under this isomorphism. For $n>1$,

$$
H^{1}\left(X ; \mathbf{Z}_{2}\right)=0 \text { and } H^{2}(X ; \mathbf{Z})=0
$$

which allows us to assert the existence of a global nowhere vanishing section $\nu$ of $N_{F}^{1 / 2}$ with $\nu \otimes \nu$ corresponding with $\mu$ under the isomorphism. We necessarily then have $\nabla^{1 / 2} \nu=\frac{1}{2} 2 \pi i n \lambda \otimes \nu=\pi i n \lambda \otimes \nu$.

We take $L^{\omega}$ as $X \times \mathbf{C}$ which has a global nowhere vanishing section $s_{0}$ given by $s_{0}(x)=(x, 1)$. Let $\alpha$ be the connection form on $L^{\omega}$ with $s_{0}^{*} \alpha=\alpha_{0}$ and let $\nabla^{\omega}$ be the corresponding connection so that $\nabla^{\omega} s_{0}=2 \pi i \alpha_{0} \otimes s_{0}$.

Setting $L=L^{\omega} \otimes N_{F}^{1 / 2}$ and $\nabla=\nabla^{\omega} \mid F \otimes 1+1 \otimes \nabla^{1 / 2}$ and $\nu_{0}=s_{0} \otimes \nu$, we have $\nabla \nu_{0}=\left(2 \pi i \alpha_{0} \mid F+\pi i n \lambda\right) \otimes \nu_{0}=(\pi i n-2 \pi i h) \lambda \otimes \nu_{0}$.Parallel-transport around the integral curve of $\xi_{h}$ through $x$ is then given by $l(x)$ $=\exp [\pi i n-2 \pi i h(x)]$. The subset $Y$ where $l(x)=1$ is $\{x \in X \mid h(x)-n / 2$ $\in \mathbf{Z}\}=\cup_{m} Y_{m}$ where $Y_{m}=h^{-1}(m+n / 2)$. Since $h$ is a positive valued function we have $m>-n / 2$ for nontrivial $Y_{m}$. 
By our result in the previous section there is an injection of $H^{p}\left(X ; \delta_{F}\right)$ into $\prod_{m \in \mathbf{Z}} H^{p}\left(Z_{m}, \delta_{\tilde{F}^{(m)}}\right)$ where $Z_{m}$ is the quotient of $Y_{m}$ by the action of $\sigma_{t}$ and $\tilde{F}^{(m)}$ the restriction of $\tilde{F}$ to $Z_{m}$. The $Z_{m}$ are the connected components of $Z$. Let $L^{(m)}$ be $\tilde{L} \mid Z_{m}$.

Now $Y_{m}=\left\{\left.\left(z_{1}, \ldots, z_{n}\right) \in \mathbf{C}^{n}\left|\pi \sum_{i=1}^{n}\right| z_{i}\right|^{2}=m+n / 2>0\right\}$ is a $2 n-1$ sphere and the action of $\sigma_{t}$ on $Y_{m}$ is the diagonal rotation of each complex variable. The map $\rho: Y_{m} \rightarrow Z_{m}$, therefore, is just the standard fibering of $S^{2 n-1}$ over $P_{n-1}(\mathrm{C})$ with fibre $S^{1} . \tilde{F}^{(m)}$ is the usual complex structure on $P_{n-1}(\mathrm{C})$ and $\tilde{L} \mid Z_{m}$ is a holomorphic line bundle. It must therefore be some power of the positive generator $\eta$ of $H^{2}\left(P_{n-1}(\mathbf{C}), \mathbf{Z}\right)$ say $L^{(m)}=\eta^{N}$. $\eta$ has transition functions $z_{j} / z_{i}$ on $U_{i} \cap U_{j}$. One easily sees this implies the pull-back $\tilde{\eta}$ of $\eta$ to $Y_{m}$ is trivial with a global section $\tilde{s}$ such that $\nabla \tilde{s}=-2 \pi i \lambda \otimes \tilde{s}$ and, hence, $\eta^{N}$ pulls back to $\tilde{\eta}^{N}$ with a section $\tilde{s}^{N}$ such that $\nabla \tilde{s}^{N}=-2 \pi i N \lambda \otimes \tilde{s}^{N}$. But $L \mid Y_{m}$ has the global section $\nu_{0}$ and $\nabla \nu_{0}=(\pi i n-2 \pi i h) \lambda \otimes \nu_{0}=-2 \pi i m \lambda$ $\otimes \nu_{0}$ on $Y_{m}$, showing $N=m$ and $L^{(m)} \cong \eta^{m}$.

In order to compute $H^{p}\left(Z_{m} ; \delta_{\tilde{F}(m)}\right)$ we use the results on the vanishing theorem of Kodaira together with Serre duality which may be found, for instance, in [3]. Kodaira's theorem implies $H^{p}\left(Z_{m}, \delta_{\tilde{F}^{(m)}}\right)=0$ for all $p$ $<n-1$ if $m<0$, whilst Serre duality applied to this result implies $H^{p}\left(Z_{m} ; \delta_{\tilde{F}(m)}\right)=0$ for all $p>0$ if $-m-n<0$, that is, $m>-n$. Thus for $m>-n / 2$ we have $H^{p}\left(Z_{m} ; \varsigma_{\tilde{F}(m)}\right)=0$ for all $p$ if $m<0$ and $H^{0}\left(Z_{m} ; \delta_{\tilde{F}(m)}\right)$ is the only nonvanishing group for $m \geqslant 0$. Its dimension is $\left(\begin{array}{c}n+m-1 \\ m\end{array}\right)$. Thus since $H^{p}\left(X ; \delta_{F}\right)$ injects into $\prod_{m>-n / 2} H^{p-1}\left(Z_{m} ; \delta_{\tilde{F}(m)}\right)$, we must have $H^{p}\left(X ; \delta_{F}\right)$ $=0$ for $p \geqslant 2$, and for $p=1$ we have an isomorphism $H^{1}\left(X ; \delta_{F}\right)$ $\cong \Pi_{m \geqslant 0} H^{0}\left(Z_{m} ; \delta_{\tilde{F}(m)}\right)$. Now $h$ is in $C_{F}(X)$, so operates on the sheaf. Under this isomorphism $h$ operates on the right-hand side also. Its value on $Y_{m}$ is $m+n / 2$ and so $h$ acts by multiplication by $m+n / 2$ on the $m$ th factor in the product. This is the correct spectrum with multiplicities for the quantum mechanical harmonic oscillator.

\section{REFERENCES}

1. R. J. Blattner, Pairing of half-form spaces, Colloque Symplectique, Aix-en-Provence, 1974.

2. S. S. Chern, Complex manifolds without potential theory, Van Nostrand Math. Studies, no. 15, Van Nostrand, Princeton, N.J., 1967. MR 37 \#940.

3. F. Hirzebruch, Topological methods in algebraic geometry, 3rd ed., Springer-Verlag, New York, 1966. MR 34 \#2573.

4. L. Hörmander, The Frobenius-Nirenberg theorem, Ark. Mat. 5 (1965), 425-432. MR 31 $\# 2480$.

5. J. B. Keller, Corrected Bohr-Sommerfeld quantum conditions for non-separable systems, Ann. Physics 4 (1958), 180-188. MR 20 \#5650.

6. B. Kostant, Quantization and unitary representations. I. Lectures in Modern Analysis and Applications. III, Lecture Notes in Math., vol. 170, Springer-Verlag, Berlin and New York, 1970. pp. 87-208. MR 45 \#3638. 
7. - Symplectic spinors, Convegno di Geomettrica Simplettica e Fisica Matematica, INDAM, Rome, 1973.

8. - On the definition of quantization, Colloque Symplectique, Aix-en-Provence, 1974.

9. H. Lewy, An example of a smooth linear partial differential equation without solution, Ann. of Math. (2) 66 (1957), 155-158. MR 19, 551.

10. L. Nirenberg, $A$ complex Frobenius theorem, Seminars on Analytic Functions. I, Princeton Univ. Press, Princeton, N.J., 1957, pp. 172-189.

11. E. Onofri and M. Pauri, Analyticity and quantization, Lett. Nuovo Cimento 3 (1972), 35-42.

12. - Dynamical quantization, J. Mathematical Phys. 13 (1972), 533-543. MR 47 \#2941.

13. E. Onofri, Quantization theory for homogeneous Kähler manifolds, Parma, 1974 (preprint).

14. P. Renouard, Variétés symplectiques et quantification, Thèse, Orsay, 1969.

15. D. J. Simms, Geometric quantization of symplectic manifolds, Internat. Sympos. Mathematical Physics, Warsaw, 1974.

16. - Geometric quantization of the harmonic oscillator with diagonalised Hamiltonian, Proc. 2nd Internat. Colloq. Group Theoretical Methods in Physics (Catholic Univ., Nijmegen, 1973).

17. - Metalinear structures and a geometric quantization of the harmonic oscillator, Colloque Symplectique, Aix-en-Provence, 1974.

18. J. Sniatycki, Bohr-Sominerfeld conditions in geometric quantization, Rep. Mathematical Phys. 7 (1974), 127-135.

19. - Bohr-Sommerfeld quantum systems, 3rd Internat. Colloq. Group Theoretical Methods in Physics (Marseille, 1974).

20. - Wave functions relative to a real polarization, Internat. J. Theoret. Phys. 14 (1975), $277-288$.

21 - On cohomology groups appearing in geometric quantization, Calgary, 1975 (preprint).

22. J.-M. Souriau, Structure des systemes dynamiques, Dunod, Paris, 1970. MR 41 \#4866.

23. F. W. Warner, Foundations of differentiable manifolds and Lie groups, Scott, Foresman, Glenview, Ill., 1971. MR 45 \#4312.

Mathematisches Institut Der Universität Bonn, Bonn, Federal Republic of Germany

Current address: School of Theoretical Physics, Dublin Institute for Advanced Studies, Dublin 4, Ireland 